\title{
Gauged merons
}

\author{
A. Samoilenka ${ }^{1}$ and Ya. Shnir ${ }^{1,2,3}$ \\ ${ }^{1}$ Department of Theoretical Physics and Astrophysics, Belarusian State University, Minsk 220004, Belarus \\ ${ }^{2}$ BLTP, JINR, Dubna 141980, Moscow Region, Russia \\ ${ }^{3}$ Department of Theoretical Physics, Tomsk State Pedagogical University, Tomsk 634061, Russia
}

(Received 6 December 2017; published 6 February 2018)

\begin{abstract}
We construct a new class of regular soliton solutions of the gauged planar Skyrme model on the target space $S^{2}$ with fractional topological charges in the scalar sector. These field configurations represent Skyrmed vortices; they have finite energy and carry topologically quantized magnetic flux $\Phi=2 \pi n$, where $n$ is an integer. Using a special version of the product ansatz as a guide, we obtain by numerical relaxation various multimeron solutions and investigate the pattern of interaction between the fractionally charged solitons. We show that, unlike the vortices in the Abelian Higgs model, the gauged merons may combine short-range repulsion and long-range attraction. Considering the strong gauge coupling limit, we demonstrate that the topological quantization of the magnetic flux is determined by the Poincare index of the planar components $\phi_{\perp}=\phi_{1}+i \phi_{2}$ of the Skyrme field.
\end{abstract}

DOI: 10.1103/PhysRevD.97.045004

\section{INTRODUCTION}

The past two decades have seen remarkable progress in our understanding of various soliton solutions in nonlinear systems. These spatially localized field configurations arise in many different areas of physics, e.g., physics of condensed matter [1,2], solid state physics [3], nonlinear optics [4], biophysics [5], field theory [6], cosmology [7], and other disciplines. This development has sparked a lot of interest in the mathematical investigation of nonlinear systems, the fascinating techniques developed in this area of modern theoretical physics, find many other applications.

An interesting example of the model, which admits soliton solutions, is the nonlinear $O(3)$ sigma model, which is also known as the baby Skyrme model. It can be considered as a planar analog of a $(3+1)$-dimensional Skyrme theory [8]. The baby Skyrme model attracts special attention since this simple theory finds various direct physical realizations. It was formulated originally as a modification of the Heisenberg model of interacting spins [9]. Further, hexagonal lattices of two-dimensional Skyrmions were observed in a thin ferromagnetic layer [10] and in a metallic itinerantelectron magnet, where the Skyrmion lattice was detected by results of neutron scattering [11]. The Skyrmions naturally arise in various condensed matter systems with intrinsic and induced chirality, and some modification of the baby Skyrme

Published by the American Physical Society under the terms of the Creative Commons Attribution 4.0 International license. Further distribution of this work must maintain attribution to the author(s) and the published article's title, journal citation, and DOI. Funded by SCOAP ${ }^{3}$. model with the Dzyaloshinskii-Moriya interaction term was suggested to model noncentrosymmetric ferromagnetic planar structures [12]. Very recently, there has been a new trend in material science: here, two-dimensional magnetic Skyrmions have been discussed in the context of future applications in development of data storage technologies and emerging spintronics; see, e.g., Refs. [13,14]. The planar Skyrmions are also known through a specific contribution to the topological quantum Hall effect [15]. In this framework, the Skyrmion-like states are coupled to fluxes of magnetic field, and they effectively represent solutions of the Skyrme-Maxwell theory.

The planar Skyrme-Maxwell model was considered for the first time in Ref. [16]. Recently, there has been renewed interest in this model related with construction of multisoliton solutions [17] and investigation of the solutions of the Bogomolny-type equation for the gauged planar Skyrme model $[18,19]$. The effect of a Chern-Simons term on the structure of the solutions of this model was studied in Refs. [20,21]. An important observation is that the magnetic flux of the solutions is not in general quantized, there is no topological number, associated with the gauge sector of the model. However, in the strong gauge coupling limit, the magnetic flux becomes quantized.

Interestingly, besides Skyrmions, the nonlinear $O(3)$ sigma model supports solutions of a different type, the merons [22]. They carry topological charge $1 / 2$; however, the merons are singular solutions, and an isolated meron has infinite energy.

The aim of the present paper is to revisit the solutions of the planar Skyrme-Maxwell theory. We introduce a new class of regular localized soliton solutions with finite 
energy, the gauged merons that are carrying topologically quantized magnetic flux and possess fractional topological charges in the scalar sector. Although these solutions resemble the vortices in the Abelian Higgs model, their properties are different; in particular, the effective potential of interaction between the gauged merons may combine a short-range monopole repulsion and a long-range dipole attraction.

\section{MODEL}

We consider the gauged planar nonlinear $O(3)$ sigma model in $(2+1)$ dimensions, defined by the Lagrangian density

$$
\begin{aligned}
L= & -\frac{1}{4 g^{2}} F_{\mu \nu} F^{\mu \nu}+\frac{1}{2} D_{\mu} \vec{\phi} \cdot D^{\mu} \vec{\phi}-\frac{1}{4}\left(D_{\mu} \vec{\phi} \times D_{\nu} \vec{\phi}\right)^{2} \\
& -V(\vec{\phi}),
\end{aligned}
$$

where the triplet of scalar fields $\vec{\phi}=\left(\phi_{1}, \phi_{2}, \phi_{3}\right)$ is constrained as $\vec{\phi} \cdot \vec{\phi}=1$ and $g$ is the gauge coupling. We introduced the usual Maxwell term with the field strength tensor defined as $F_{\mu \nu}=\partial_{\mu} A_{\nu}-\partial_{\nu} A_{\mu}$. The coupling of the Skyrme field to the magnetic field is given by the covariant derivative $[16,18,23]$

$$
D_{\mu} \vec{\phi}=\partial_{\mu} \vec{\phi}+A_{\mu} \vec{\phi} \times \vec{n} \quad \vec{n}=(0,0,1) .
$$

Note that the potential breaks the original $O(3)$ symmetry of the sigma model to $O(2)$, and the Lagrangian (1) is invariant under the local $U(1)$ transformations

$$
\phi_{\perp} \rightarrow e^{i \alpha} \phi_{\perp}, \quad A_{\mu} \rightarrow A_{\mu}+\partial_{\mu} \alpha_{\mu},
$$

where $\phi_{\perp}=\phi_{1}+i \phi_{2}$. Henceforth, we consider only static configurations with $A_{0}=0$, with magnetic field $B=$ $\partial_{1} A_{2}-\partial_{2} A_{1}$.

In $2+1$ dimensions, the presence of the potential term $V(\vec{\phi})$ in (1) is necessary for the stability of the solitons. On the other hand, the structure of the potential is critical for the properties of multisoliton solutions of the model; it defines the vacuum of the model and the asymptotic behavior of the fields.

The most common choice is to consider potentials with a discrete number of isolated vacua; in the simplest case, there is a single vacuum at $\phi_{3}=1$ [16]. Other possibilities include the double vacuum potential [23], triple vacuum potential [24], or easy plane potential, which vanishes at the equator of the target space $S^{2}[25,26]$.

Here, we consider the planar Maxwell-Skyrme model with more general symmetry breaking potential,

$$
V(\vec{\phi})=\frac{1}{2} m^{2}\left(\phi_{3}-c\right)^{2},
$$

where $c \in[-1,1]$. A particular choice $c=0$ reduces the model to the gauged theory with the easy plane potential, while setting $c= \pm 1$ yields the vacuum on the north/south poles of the target space, respectively. In the ungauged model with such a potential, the asymptotic value of the fields breaks the residual $S O(2)$ internal symmetry, so the field has only discrete symmetry, and a unit charge Skyrmion is a bound state of two half-lumps; however, the total charge of the configuration remains integer valued [27].

The situation changes radically when the system is coupled to the gauge field, since the vacuum $\phi_{3}=c$ corresponds to a loop on the surface of the target space $S^{2}$.

The finiteness of the energy of the model (1) in particular implies that the magnetic field must asymptotically vanish; it corresponds to the pure gauge vacuum on the boundary $S^{1}$. On the other hand, the vacuum boundary condition implies that $\phi_{3}=c$ as $r \rightarrow \infty$ and $D_{i} \phi_{\perp}=$ $\partial_{i} \phi_{\perp}-i A_{i} \phi_{\perp} \underset{r \rightarrow \infty}{\rightarrow} 0$. This yields

$\phi_{\perp} \underset{r \rightarrow \infty}{\rightarrow} \sqrt{1-c^{2}} e^{i \Psi(\theta)}, \quad A_{i} \underset{r \rightarrow \infty}{\rightarrow} \partial_{i} \alpha(\theta), \quad \theta \in[0,2 \pi]$.

We thus obtain on the boundary $\partial_{i} \Psi(\theta)=A_{i}(\theta)$ and $\Psi(\theta)=\alpha(\theta)-\kappa$, where $\kappa$ is an angle of orientation of the configuration. Using these boundary conditions and the Stokes theorem, we can see that the magnetic flux is topologically quantized; the total phase winding is

$$
\Phi=\oint_{S^{1}} A_{i} d x^{i}=\oint_{S^{1}} \partial_{i} \alpha d x^{i}=2 \pi n,
$$

where $n \in \mathbb{Z}$. Hence, the model (1) supports topological solitons, classified by the first homotopy group $\pi_{1}\left(S^{1}\right)$. The corresponding invariant $n$ is given by the mapping of the spacial boundary $S^{1}$ onto the vacuum, which also represents a loop on the target space. Note that this invariant is exactly the Poincare index of the planar components $\phi_{\perp}$, which possesses a zero as $\phi_{3}= \pm 1$. This point corresponds to the location of the soliton coupled to the magnetic flux.

A peculiar feature of these configurations is that, since in the vacuum $\phi_{3}=c$, the topological charge in the scalar sector is no longer an integer. Indeed, the degree of the map is

$$
Q=-\frac{1}{4 \pi} \int d^{2} x \vec{\phi} \cdot\left(\partial_{1} \vec{\phi} \times \partial_{2} \vec{\phi}\right)
$$

and assuming that at the origin $\vec{\phi}(0)=(0,0,-1)$, we obtain in the simplest case $Q=(1+c) / 2$. Alternatively, as $\vec{\phi}(0)=(0,0,1)$, we obtain $Q=(1-c) / 2$; in particular, setting $c=0$ yields two solutions with half-integer scalar charge. Note that in the usual $O(3)$ sigma model the localized Euclidean configurations with a half-unit of topological charge are known as merons [22]; however, 


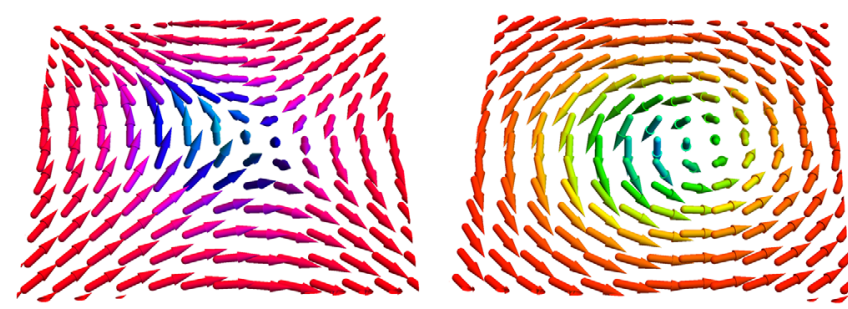

FIG. 1. The isovector fields $\vec{\phi}$ of the $(1 / 2)_{N}$ (left plot) and $(1 / 2)_{S}$ (right plot) gauged merons in the $x-y$ plane for $g=0.5$, $m=1$.

they are singular. Similar fractionally charged self-dual vortex solutions also exist in the $\mathcal{N}(2,2)$ supersymmetric gauged $\mathbb{C} P^{1}$ model [28] and in the chiral magnetic systems with external magnetic field [29].

The two meron solutions above are topologically different; thus, in the former case, the field configuration will be denoted as $k(Q)_{S}$, while in the latter, it is $k(Q)_{N}$, with the $S$ and $N$ merons wrapping lower and upper domains of the target space, respectively-see Fig. 1. Here, the integer $k$ is the number of the merons of a given type, the Poincare index $n=k$ for $S$ merons and $n=-k$ for $N$ merons. The magnetic flux of the $k(Q)_{S}$ configuration is directed along the positive direction of the $z$ axis. It is reflected for the $k(1 / 2)_{N}$ meron. However, the energy density distributions of both merons are identical. ${ }^{1}$

We can now construct gauged merons numerically. The field equations of the model (1) can be written in the form

$$
\left\{\begin{array}{l}
D_{\mu} \vec{J}^{\mu}+m^{2}(\vec{\phi} \cdot \vec{n}-c)(\vec{\phi} \times \vec{n})=0 \\
\partial_{\mu} F^{\mu \nu}-g^{2} \vec{n} \cdot \vec{J}^{\nu}=0
\end{array},\right.
$$

where the current is defined as

$$
\vec{J}^{\mu}=\vec{\phi} \times D^{\mu} \vec{\phi}-D_{\nu} \vec{\phi}\left(\vec{\phi} \cdot D^{\mu} \vec{\phi} \times D^{\nu} \vec{\phi}\right) .
$$

For the sake of simplicity, we set $c=0$. It yields two types of solutions $n(1 / 2)_{N, S}$. In our numerical simulations, we start from an initial field configuration for an $(1 / 2)_{S}$ meron, which is produced by the rotationally invariant ansatz in polar coordinates in the $x-y$ plane,

$$
\begin{aligned}
\vec{\phi} & =(\sin f \cos n \theta, \sin f \sin n \theta, \cos f), \quad A_{r}=0, \\
A_{\theta} & =A(r),
\end{aligned}
$$

where $f(r) \in[\pi, \pi / 2)$. An input for a multimeron configuration can be constructed via the product ansatz in

\footnotetext{
${ }^{1}$ Note that for an $S / N$ meron there exists an antimeron $\bar{S} / \bar{N}$ with opposite sign for both $Q$ and $n$, so an $S$ meron is not an antimeron with respect to an $N$ meron, and visa versa. For $c=0$, all four of these merons have the same energy and the magnitude of the magnetic fluxes.
}

stereographic notation; for example, the two-meron configuration corresponds to

$$
W^{(1+2)}=W^{(1)} W^{(2)} \sqrt{\frac{1+c}{1-c}}, \quad A_{i}^{(1+2)}=A_{i}^{(1)}+A_{i}^{(2)},
$$

where $W=\frac{\phi_{\perp}}{1+\phi_{3}}$. However, in our calculations, we do not adopt any a priori assumptions about spatial symmetries of components of the field configuration. In particular, the scalar field of the initial two-meron configuration constructed via the ansatz (8), for $c=0$, can be written as

$\phi_{\perp}^{(1+2)}=\frac{\phi_{\perp}^{(1)} \phi_{\perp}^{(2)}}{1+\phi_{3}^{(1)} \phi_{3}^{(2)}}, \quad \phi_{3}^{(1+2)}=\frac{\phi_{3}^{(1)}+\phi_{3}^{(2)}}{1+\phi_{3}^{(1)} \phi_{3}^{(2)}}$.

As is well known, the asymptotic behavior of the scalar and magnetic fields almost completely determines the character of interaction between the solitons $[16,25,30]$. Note that the rotational invariance of an isolated meron together with the gauge invariance with respect to the transformations (2) and asymptotic boundary conditions (3) imply that a spacial rotation of the configuration can always be compensated by an appropriate gauge transformation. In other words, the asymptotic form of the planar components of the scalar field $\phi_{\perp}$ and relative orientation of the solitons does not play a special role in the pattern of interaction between the gauged merons.

Linearization of the field equations (5) yields

$$
\left\{\begin{array}{l}
\left(\Delta-m^{2}\right) \phi_{3}=0 \\
\left(\Delta-g^{2}\right) \delta A_{i}=0 \\
\partial_{i} \delta A_{i}=0
\end{array}\right.
$$

thus, the fields of the meron decay asymptotically as

$$
\phi_{3}(r) \sim c_{s} K_{0}(m r), \quad A_{\theta}(r) \sim n+c_{v} r K_{1}(g r),
$$

where $K_{i}$ are $i$ th modified Bessel functions of the second kind and $c_{s}$ and $c_{v}$ are two constants that can be evaluated numerically. In particular, we found that for the $(1 / 2)_{S}$ configuration (7) at $m=1$ and $g \in[0,1.5]$ these parameters are $c_{v} \simeq-1$ and $c_{s} \in[-3.5,-1.6]$. Below, we will make use of these values to evaluate the net force of the interaction between the gauged merons; see Fig. 2.

Note that both fields are massive and have a form of scalar monopole and vector dipole with a fixed phase, $\delta \vec{A}=c_{v} \nabla \times \vec{n} K_{0}(g r)$. In the decoupling limit $g \rightarrow 0$, one of the components of the scalar field remains massless, $\sim d / r$, and in the far field limit, it corresponds to a source with dipole strength $d$ [25].

Using asymptotic (11) and considering the two meron configuration (8), we can evaluate the potential energy of 

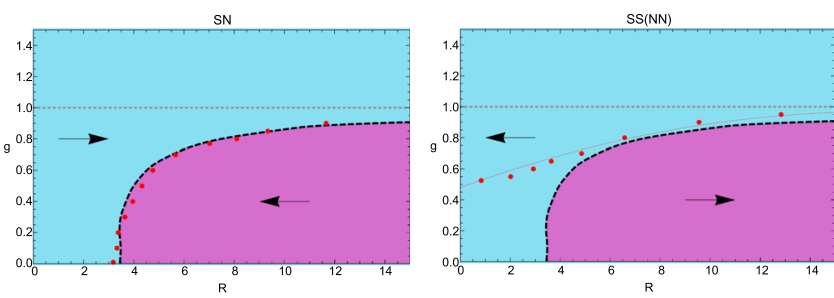

FIG. 2. Interaction of the gauged merons in the $S N$ pair (left) and in the $N N(S S)$ pair (right). Arrows show the direction of the force. The blueish area corresponds to the scalar fielddomination region, and the purple area represents the vector field-domination region. The black dashed line indicates the equilibrium curve $F=0$, and the red dots indicate the numerical solutions of full two-dimensional minimization of the static energy of the system (1).

the short-range Yukawa interaction between two static separated merons $U_{\text {int }}=E^{(1+2)}-E^{(1)}-E^{(2)}$.

Let us consider field of two widely separated merons in the vicinity of the first soliton. Then, setting $c=0$ gives $\phi_{3}^{(2)} \rightarrow 0$, and from (9) and (8) we obtain

$$
\left\{\begin{array}{l}
\phi_{\perp}^{(1+2)^{\prime}}=\phi_{\perp}^{(1)} \phi_{\perp}^{(2)} \\
\phi_{3}^{(1+2)^{\prime}}=\phi_{3}^{(1)} \\
A_{i}^{(1+2)^{\prime}}=A_{i}^{(1)}+A_{i}^{(2) \infty}
\end{array} .\right.
$$

However, the field of the second meron here has the form (3) i.e., up to the corresponding gauge transformation, we can write $\phi_{\perp}^{(1+2)^{\prime}}=\phi_{\perp}^{(1)}, A_{i}^{(1+2)^{\prime}}=A_{i}^{(1)}$. Then, expanding (9) up to first order in $\phi_{3}^{(2)}$, we get

$$
\left\{\begin{array}{l}
\vec{\phi}^{(1+2)}=\vec{\phi}^{(1+2)^{\prime}}+\vec{\epsilon}^{(2)} \times \vec{\phi}^{(1+2)^{\prime}} \\
A_{i}^{(1+2)}=A_{i}^{(1+2)^{\prime}}+\delta A_{i}^{(2)}
\end{array},\right.
$$

where $\vec{\epsilon}^{(2)}=\phi_{3}^{(2)}\left(\vec{\phi}^{(1+2)^{\prime}} \times \vec{n}\right)$. This expansion can be used to evaluate the potential energy of interaction between the solitons following [31]:

$$
U_{\mathrm{int}}=2 \pi\left(c_{v}^{(1)} c_{v}^{(2)} K_{0}(g r)-c_{s}^{(1)} c_{s}^{(2)} K_{0}(m r)\right) .
$$

This formula exactly corresponds to the asymptotic intervortex potential in the Abelian Higgs model [30]; however, the character of interaction depends on the type of the solitons. The force between the merons can be evaluated as

$F=-U_{\mathrm{int}}^{\prime}= \pm 2 \pi c_{s}^{2} m\left(\eta^{2} g / m K_{1}(g R)-K_{1}(m R)\right)$,

where $\eta=c_{v} / c_{s}$ and the sign " + " corresponds to the interaction between the merons of the same type in the $N N$ pair (or in the $S S$ pair). The opposite sign corresponds to the interaction between the merons of different types; they form the $N S$ pair.
Next, for each particular value of the gauge coupling $g$, we can evaluate the separation $R_{0}: F\left(R_{0}\right)=0$, at which the forces between the merons are balanced. We expect that there will be a stable equilibrium for the system of two merons of different types, $N$ and $S$, whereas the interforce balance between the pair of $S$ or $N$ merons will be unstable. Note that, unlike the case of interaction between the vortices in the Abelian Higgs model [30], for $g / m<1$ the $N N$ (or $S S$ ) merons with relatively small initial separation merge, forming a rotationally invariant configuration with multiple magnetic flux; see Fig. 2 right plot.

We numerically minimized full two-dimensional energy functional without any restrictions on the symmetry or gauge on $A_{i}$. Typically, we used a lattice with $200 \times 200$ grid points and spacing $d x=0.5$, and the algorithm was based on the one described in Ref. [17]. The results of numerical simulations are summarized in Fig. 2; there, without loss of generality, we fix $m=1$. We confirm that the approximation of the intersoliton force (15) works very well. It correctly predicts the separation between the $N$ and $S$ merons in a stable equilibrium; see Fig. 2, left plot. Remarkably, the pair does not form a rotationally invariant configuration for any values of the gauge coupling; there is a short-range repulsive force between the merons of different types, and the NS pair remains separated.

The equilibrium between the merons of the same type is unstable. Furthermore, in the case of the weak gauge coupling, the separation between the merons becomes rather small, and the asymptotic evaluation above breaks down; see Fig. 2, right plot.

\section{MULTIMERON CONFIGURATIONS}

We observe that as $g \gtrsim 1$ the $N N(S S)$ pair always tends to merge into a rotationally invariant configuration with double magnetic flux for any initial separation between the merons. More generally, in the strong coupling regime, the system of $n$ separated gauged merons of the same type evolves toward a rotationally invariant configuration with $n$ units of magnetic flux. In Fig. 3, we present the results of the full numerical minimization of the energy functional for the $n(1 / 2)_{S}$ configurations with $n=1-4$. As expected, the field components become less localized, and the core of the vortex is expanding, as the winding number $n$ increases. The energy density distribution of the $n=1$ configuration reaches its maximum value at the center of the soliton; for $n>1$, it has the shape of a circular wall with a local minimum at the origin. Field components of these solutions along the $x$ axis are displayed in Fig. 3 (top row), and both $\phi_{1}$ and $\phi_{3}$ decay exponentially; however, at $g=4$, the former component approaches the vacuum faster than the latter.

Evaluation of the intersoliton forces above indicates that for $m=1$ and $0.57 \lesssim g<1$ we could construct stable multisoliton configuration with merons of both types. Indeed, it is seen in Fig. 4, which displays contour plots 

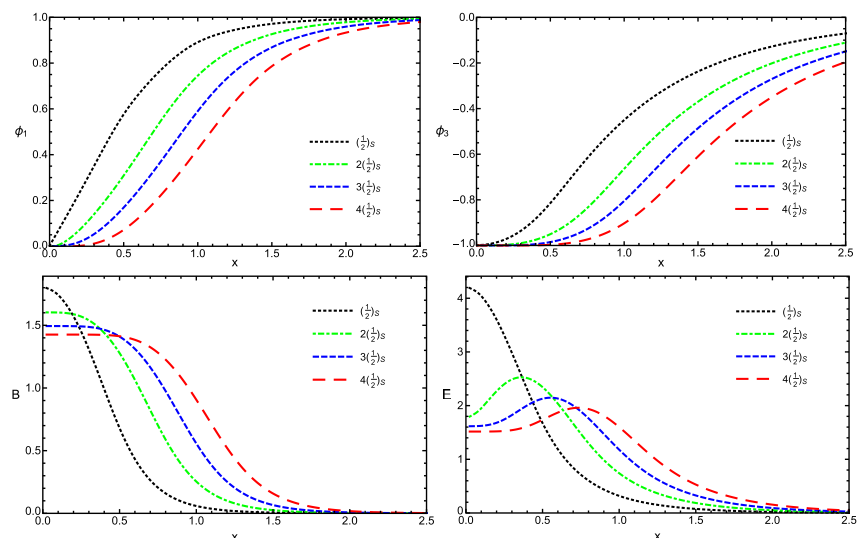

FIG. 3. Rotationally invariant $n(1 / 2)_{S}$ configurations: Profiles of the field components $\phi_{1}$ (upper left) and $\phi_{3}$ (upper right), the distributions of the magnetic field (bottom left) and the energy density (bottom right) along the $x$-axis for $n=1-4, g=4$ and $m=1$.

of the magnetic field and the energy density distribution of various solutions that we constructed numerically, that in such a case the rotational symmetry becomes broken and the gauged merons form configurations with discrete symmetry.

Note that the $(1 / 2)_{S}+(1 / 2)_{N}$ pair combines a shortrange repulsion and a long-range attraction, forming a weakly bound system. Certainly, there is a similarity with the aloof baby Skyrmions constructed in Ref. [32]. Further, the binary particle model suggested in Ref. [32] also can be implemented in the case of the gauged multimeron configurations.

Our numerical results presented in Fig. 4 agree well with the qualitative discussion of the intersoliton interaction above, see Fig. 2. We observe that, as the separation between the merons of the same type is relatively small, they tend to merge into a symmetric configuration that carries multiple magnetic flux. On the other hand, widely separated merons repel each other. The energy per meron is decreasing as the number of components is increasing; thus, the system is stable with respect to decay into constituents. Also, the configurations with constituents possessing multiple units of magnetic flux, for example, $(1 / 2)_{N}+2(1 / 2)_{S}$, have lower energy than the chain $(1 / 2)_{N}+(1 / 2)_{S}+(1 / 2)_{N}$. The latter configuration represents a local minimum of the energy functional.

Finally, we would like to comment on the limit of the single vacuum potential. Setting $c= \pm 1$ reduces it to $V(\vec{\phi})=m^{2}\left(1 \mp \phi_{3}\right)^{2}$. In this limit, the magnetic flux is no longer topologically quantized. However, numerical simulations show that in the strong gauge coupling limit it becomes quantized again $[16,17]$. Numerical simulations reveal that the maxima of the magnetic field correspond to the points where $\phi_{3}= \pm 1$; see Fig. 4 . This observation holds for any values of the parameter $c$ and different multisoliton configurations, for example, for individual separated merons shown in Fig. 4. In the limit $g \rightarrow \infty$,
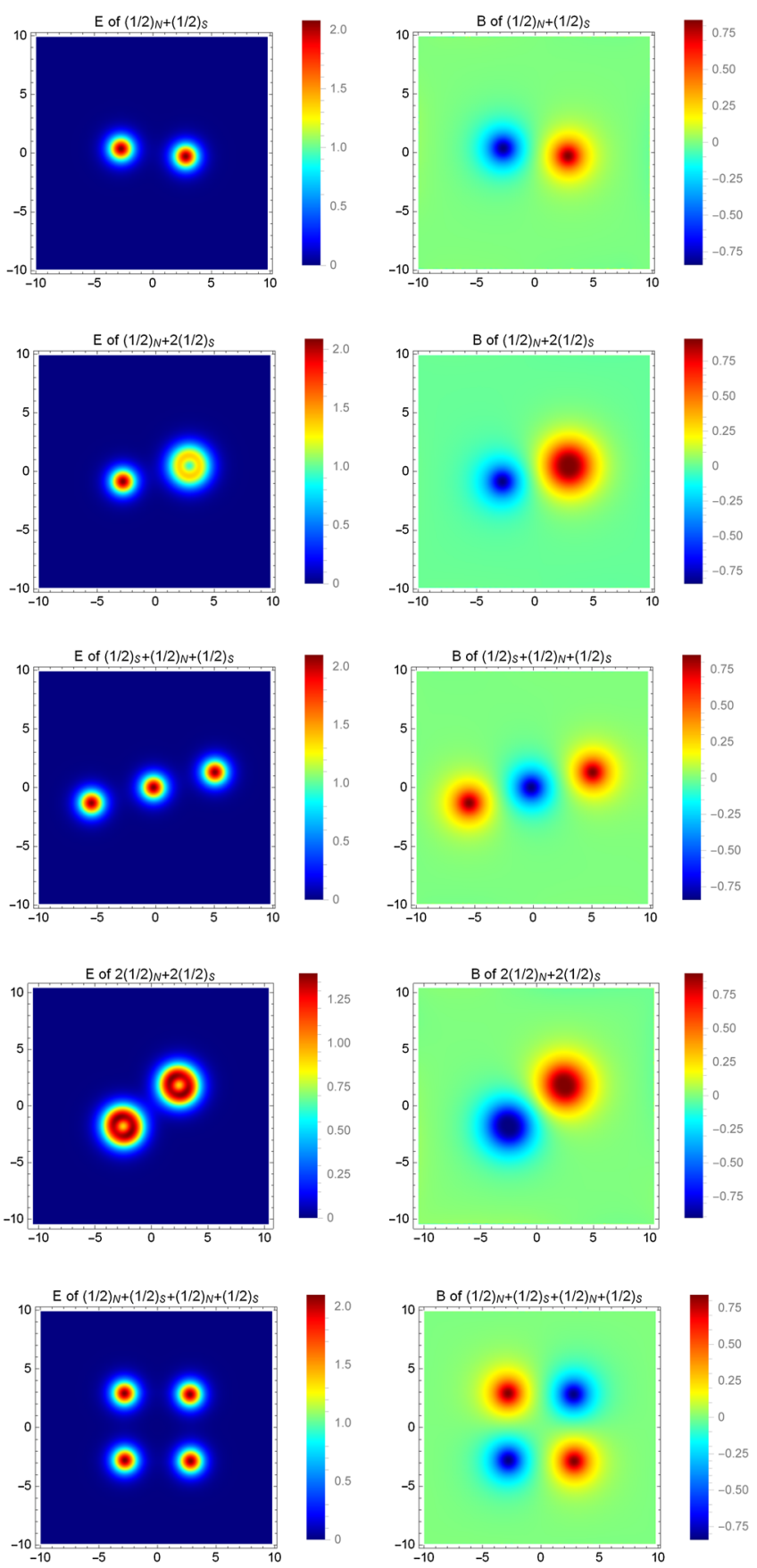

FIG. 4. Contour plots of the energy density (left column) and magnetic field (right column) of given meron configurations at $g=0.7, m=1$.

the magnetic field becomes completely localized at the $\phi_{3}= \pm 1[16]$.

We can understand the underlying topological reason for this when we consider the Maxwell equation, which corresponds to the second of the field equations (5). The electromagnetic current, which is a source for the magnetic flux, is $j_{i}=\vec{n} \cdot\left[\vec{\phi} \times D_{i} \vec{\phi}+D_{j} \vec{\phi}\left(\vec{\phi} \cdot D_{i} \vec{\phi} \times D_{j} \vec{\phi}\right)\right]$. As $g \rightarrow \infty$, we can see that $j_{i}=-1 / g^{2} \partial_{k} F_{k i}$; thus, $j_{i}=0$ apart from some set of isolated points, where $A_{\theta}$ changes quickly 
from $n$ to 0 . Effectively, in such a limit, the magnetic energy is removed from the system (1). Further, we can simplify the expression for the current introducing the ratio of the planar components of the scalar field, $\sigma=\arctan$ $\left(\phi_{2} / \phi_{1}\right)$ :

$$
\begin{aligned}
j_{i}= & \left(\partial_{i} \sigma-A_{i}\right)\left[1-\phi_{3}^{2}+\partial_{j} \phi_{3}^{2}\right] \\
& -\partial_{i} \phi_{3} \partial_{j} \phi_{3}\left(\partial_{j} \sigma-A_{j}\right)=0 .
\end{aligned}
$$

Evidently, the potential of the gauge field then becomes a pure gauge, $A_{i}=\partial_{i} \sigma$, everywhere apart from the points where $\phi_{3}= \pm 1$, and the magnetic flux is entirely determined by the Poincaré index of the planar components $\phi_{\perp}$. A similar pattern also holds for the gauged Hopfion solutions in the Faddeev-Skyrme model [33].

\section{CONCLUSIONS}

Our investigation confirms the existence of a new type of regular finite energy solutions of the planar MaxwellSkyrme model, the gauged merons. They carry topologically quantized magnetic flux and possess fractional topological charges in the scalar sector. The vortex winding number is set into correspondence with the Poincare index of the planar components of the meron. Considering the interaction between the gauged merons, we have shown that, unlike the usual vortices in the Abelian Higgs model, they may combine a short-range repulsion and a long-range attraction, forming a weakly bound nonrotationally invariant system. The resulting pattern of interaction is more complicated than that both for the usual vortices in the Abelian Higgs model and for the solitons in the gauged baby Skyrme model. It remains a major challenge, deserving further study, to find a moduli space description for the low-energy dynamics of the gauged merons.

\section{ACKNOWLEDGMENTS}

A. S. is very grateful to the organizers, lecturers, and participants of the MITP Summer School 2017, where this work was initiated, for many stimulating discussions and especially to Professor David Morrissey, whose lectures served as inspiration. Y. S. thanks Nick Manton, Muneto Nitta, and Nobuyuki Sawado for helpful discussions. He gratefully acknowledges support from the Russian Foundation for Basic Research (Grant No. 16-52-12012), the Ministry of Education and Science of Russian Federation (Project No. 3.1386.2017), and DFG (Grant No. LE 838/12-2). The numerical computations were performed on the HYBRILIT cluster, JINR, Dubna.
[1] Solitons and Condensed Matter Physics, edited by A. R. Bishop and T. Schneider (Springer-Verlag, Berlin, 1978).

[2] P. J. Ackerman and I. I. Smalyukh, Phys. Rev. X 7, 011006 (2017).

[3] A. M. Kosevich, The Crystal Lattice: Phonons, Solitons, Dislocations, Superlattices (Wiley, New York, 2006).

[4] L. F. Mollenauer and J.P. Gordon, Solitons in Optical Fibers (Academic, New York, 2006).

[5] T. Dauxois and M. Peyrard, Physics of Solitons (Cambridge University Press, Cambridge, 2006); M. Peyrard, Nonlinear Excitations in Biomolecules (Springer-Verlag, Berlin, 1995).

[6] N. Manton and P. Sutcliffe, Topological Solitons (Cambridge University Press, Cambridge, England, 2004).

[7] A. Vilenkin and E.P.S. Shellard, Cosmic Strings and Other Topological Defects (Cambridge University Press, Cambridge, 1994).

[8] T. H. R. Skyrme, Proc. R. Soc. A 260, 127 (1961).

[9] A. A. Bogolubskaya and I. L. Bogolubsky, Phys. Lett. A 136, 485 (1989).

[10] X. Z. Yu, Y. Onose, N. Kanazawa, J. H. Park, J. H. Han, Y. Matsui, N. Nagaosa, and Y. Tokura, Nature (London) 465, 901 (2010).

[11] S. Mühlbauer, B. Binz, F. Jonietz, C. Pfleiderer, A. Rosch, A. Neubauer, R. Georgii, and P. Boni, Science 323, 915 (2009).
[12] A. N. Bogdanov and D. A. Yablonskii, Zh. Eksp. Teor. Fiz 95, 182 (1989); A. Bogdanov, JETP Lett. 62, 247 (1995).

[13] S. Heinze, K. von Bergmann, M. Menzel, J. Brede, A. Kubetzka, R. Wiesendanger, G. Bihlmayer, and S. Blügel, Nat. Phys. 7, 713 (2011).

[14] Skyrmions: Topological Structures, Properties, and Applications, edited by J. P. Liu, Z. Zhang, and G. Zhao (CRC Press, Boca Raton, 2016).

[15] A. Neubauer, C. Pfleiderer, B. Binz, A. Rosch, R. Ritz, P. G. Niklowitz, and P. Böni, Phys. Rev. Lett. 102, 186602 (2009).

[16] J. Gladikowski, B. M. A. G. Piette, and B. J. Schroers, Phys. Rev. D 53, 844 (1996).

[17] A. Samoilenka and Y. Shnir, Phys. Rev. D 93, 065018 (2016).

[18] C. Adam, C. Naya, J. Sanchez-Guillen, and A. Wereszczynski, Phys. Rev. D 86, 045010 (2012).

[19] C. Adam and A. Wereszczynski, Phys. Rev. D 95, 116006 (2017).

[20] F. Navarro-Lerida, E. Radu, and D. H. Tchrakian, Phys. Rev. D 95, 085016 (2017).

[21] A. Samoilenka and Y. Shnir, Phys. Rev. D 95, 045002 (2017).

[22] D. J. Gross, Nucl. Phys. B132, 439 (1978).

[23] B. J. Schroers, Phys. Lett. B 356, 291 (1995). 
[24] P. Eslami, M. Sarbishaei, and W. Zakrzewski, Nonlinearity, 13, 1867 (2000).

[25] J. Jaykka and M. Speight, Phys. Rev. D 82, 125030 (2010).

[26] M. Kobayashi and M. Nitta, Phys. Rev. D 87, 125013 (2013).

[27] Y. Brihaye, B. Hartmann, and D. H. Tchrakian, J. Math. Phys. 42, 3270 (2001).
[28] M. Nitta and W. Vinci, J. Phys. A 45, 175401 (2012).

[29] S. Z. Lin, A. Saxena, and C. D. Batista, Phys. Rev. B 91, 224407 (2015).

[30] J. M. Speight, Phys. Rev. D 55, 3830 (1997).

[31] B. M. A. G. Piette, B. J. Schroers, and W. J. Zakrzewski, Z. Phys. C 65, 165 (1995).

[32] P. Salmi and P. Sutcliffe, J. Phys. A 48, 035401 (2015).

[33] Y. Shnir and G. Zhilin, Phys. Rev. D 89, 105010 (2014). 